\title{
Urinary expression of long non-coding RNA TUG1 in non-diabetic patients with glomerulonephritides
}

\author{
FERNANDO JAVIER SALAZAR-TORRES ${ }^{1,2}$, MIGUEL MEDINA-PEREZ ${ }^{1}$, ZESERGIO MELO $^{3}$, \\ CLAUDIA MENDOZA-CERPA ${ }^{4}$ and RAQUEL ECHAVARRIA ${ }^{3}$ \\ ${ }^{1}$ Departamento de Nefrología, UMAE-Hospital de Especialidades, \\ CMNO, Instituto Mexicano del Seguro Social, Guadalajara, Jalisco 44340; \\ ${ }^{2}$ Unidad de Medicina Familiar con Unidad Médica de Atención Ambulatoria UMF/UMAA 39, \\ Instituto Mexicano del Seguro Social, Matamoros, Tamaulipas 87344; \\ ${ }^{3}$ CONACyT-Centro de Investigación Biomédica de Occidente, Instituto Mexicano del Seguro Social; \\ ${ }^{4}$ Departamento de Patología, UMAE-Hospital de Especialidades, \\ CMNO, Instituto Mexicano del Seguro Social, \\ Guadalajara, Jalisco 44340, México
}

Received March 4, 2020; Accepted September 29, 2020

DOI: $10.3892 /$ br.2020.1393

\begin{abstract}
Metabolic alterations serve a significant role in the pathogenesis of kidney disease. Long non-coding RNA (lncRNA) taurine upregulated gene 1 (TUG1) is a known regulator of podocyte health and mitochondrial biogenesis. Although TUG1 protects against podocyte loss in models of diabetic nephropathy, it is unknown if urinary TUG1 expression is associated with clinical and histopathological findings in non-diabetic patients diagnosed with glomerulonephritides. In the present study, the expression of TUG1, podocyte-specific markers (nephrin and podocin) and mitochondrial biogenesis-associated mRNAs (transcription factor A mitochondrial, cytochrome $\mathrm{C}$ oxidase subunit $5 \mathrm{~A}$ and peroxisome proliferator-activated receptor $\gamma$ coactivator $1 \alpha$ ) were examined in urinary sediment of non-diabetic patients
\end{abstract}

Correspondence to: Dr Raquel Echavarria, CONACyT-Centro de Investigación Biomédica de Occidente, Instituto Mexicano del Seguro Social, Sierra Mojada 800 Col. Independencia, Guadalajara, Jalisco 44340, México

E-mail: rechavarria@conacyt.mx

Abbreviations: IncRNA, long non-coding RNA; TUG1, taurine upregulated gene 1; TFAM, transcription factor A mitochondrial, COX5A, cytochrome $\mathrm{C}$ oxidase subunit 5A; and PPARGC1A, peroxisome proliferator-activated receptor $\gamma$ coactivator $1 \alpha$; ESRD, end-stage renal disease; FSGS, focal segmental glomerulosclerosis; DN, diabetic nephropathy; AGS, advanced glomerular sclerosis; NSPH1, nephrin; NSPH2, podocin; GAPDH, glyceraldehyde-3-phosphate dehydrogenase; LN, lupus nephritis; AAV, ANCA-associated vasculitis; GFR, glomerular filtration rate

Key words: TUG1, lncRNA, glomerulonephritides, FSGS, urinary sediment with biopsy-confirmed glomerulonephritides and healthy controls. Urinary expression of TUG1 was significantly lower in patients with glomerulonephritides, particularly those diagnosed with Focal Segmental Glomerulosclerosis (FSGS). Furthermore, TUG1 levels were associated with urinary expression of podocyte-specific markers and mRNAs associated with mitochondrial biogenesis. Loss of TUG1 expression in urinary sediment was strongly associated with FSGS, highlighting the potential of this lncRNA and its mitochondrial biogenesis-associated targets as non-invasive biomarkers of assessing podocytopathy.

\section{Introduction}

Glomerulonephritides are a group of rare diseases that often affects younger individuals and can lead to end-stage renal disease (ESRD) (1). Although the clinical presentation of glomerulonephritides and their outcomes are variable, well-established factors such as persistent proteinuria, hypertension, diabetes and cardiovascular disease increase the risk of ESRD progression (2). Podocyte injury is a hallmark of renal diseases, presenting clinically with proteinuria, glomerulosclerosis and kidney failure (3). Terminally differentiated podocytes have a limited capacity to self-replicate; hence, direct cellular damage contributes to the onset and progression of glomerular diseases such as Focal Segmental Glomerulosclerosis (FSGS), Minimal Change Disease and Diabetic Nephropathy (DN) (4). Additionally, immune-mediated damage is responsible for the establishment and progression of secondary glomerulonephritides, including lupus nephritis (LN) and ANCA-associated vasculitis (AAV) (5).

The gold standard for confirming a diagnosis of glomerulonephritides diagnosis is renal biopsy (6). However, biopsies are invasive, may have complications and are hard to interpret due to the presence of highly complex pathogenic mechanisms that translate into limited histological responses of kidney injury (7). Thus, identifying non-invasive biomarkers 
of glomerular disease may result in improved diagnosis and guide therapeutic choices in the field of nephrology (8).

Long non-coding RNAs (lncRNAs) are RNA molecules comprised of $>200$ nucleotides with no protein-coding capacity. They are a class of RNAs comprised of heterogeneous intergenic transcripts, sense or antisense transcripts that overlap with other genes, or enhancer RNAs with a range of functions that includes the regulation of gene expression, chromatin remodelling, microRNA (miRNA/miR)-sponging and protein scaffolding $(9,10)$. LncRNAs modulate several biological processes, including homeostasis, cellular metabolism, proliferation, apoptosis and differentiation $(10,11)$. Dysregulated expression of 1ncRNAs such as metastasis-associated lung adenocarcinoma transcript 1 , LOC105374325, LOC105375913, X-inactive specific transcript and RP11-2B6.2 contribute to the pathogenesis of various kidney diseases (12-17). Moreover, circulating lncRNAs are stable and easily detectable in plasma, serum and urine, characteristics that make them convenient for use diagnostically $(18,19)$.

The IncRNA taurine upregulated gene 1 (TUG1), located on chromosome $22 \mathrm{q} 12$, regulates podocyte health and glomerulosclerosis by altering the expression of peroxisome proliferator-activated receptor $\gamma$ Coactivator $1 \alpha$ (PGC1A) (20-23). PGC1A is a transcriptional coactivator that controls mitochondrial biogenesis and is encoded by the PPARGC1A gene in humans $(24,25)$. A decrease in PGC1A expression contributes to the onset of metabolic diseases such as DN, and transgenic expression of PPARGC1A in tubular cells protects mice from developing acute and chronic forms of kidney disease $(26,27)$. Long et al (28) found that podocyte-specific transgenic expression of lncRNA TUG1 protects against $\mathrm{DN}$ in a mouse model, and demonstrated that lower glomerular filtration rates (GFR) were correlated with a decrease in TUG1 intrarenal expression in human subjects. However, it is unknown if TUG1 can be detected in urine and if its expression levels are correlated with histopathological findings in kidney biopsies from patients diagnosed with glomerulonephritides other than DN. In the present study, the urinary expression of TUG1 in non-diabetic patients with glomerulonephritides was characterized and it was shown that a decrease in TUG1 expression was significantly associated with FSGS.

\section{Materials and methods}

Ethical considerations. The present study complied with the ethical principles for medical research specified in the Declaration of Helsinki (29) and was approved by the Local Ethics and Research Committee at the Hospital de Especialidades NUM. 1, Bajio, Leon, Guanajuato, Instituto Mexicano del Seguro Social (approval no. CLIEIS R-2018-1001-114). Each participant provided written informed consent prior to enrolment in the study.

Patient enrolment. A total of 11 patients with biopsy-confirmed glomerulonephritides ( 7 females and 4 males; median age, 31 years; range $19-59$ years), and 10 healthy controls (6 females and 4 males; median age 34 years; range 22-54) were enrolled in the present study at UMAE-Hospital de Especialidades, CMNO in Guadalajara, Mexico. Inclusion criteria were non-diabetic patients aged $\geq 18$ years. Exclusion criteria were intake of non-steroid anti-inflammatory drugs or platelet anti-aggregation medication a week before sampling, administration of anticoagulant medication $96 \mathrm{~h}$ before the biopsy, blood pressure $>160 / 90 \mathrm{mmHg}$, active urinary infection or anaemia. Urine sample collection was taken shortly before biopsy collection. Only 1 patient enrolled in the study was unable to provide a urine sample and, therefore, was excluded from further analysis.

Data collection. Biochemical parameters corresponding to each glomerulonephritides patient at the time of biopsy were assayed by routine laboratory techniques at UMAE-Hospital de Especialidades and retrieved from patients' electronic medical records.

Reverse transcription-quantitative $(R T-q) P C R$. Urine specimens from patients and healthy controls were collected and centrifuged at $4,500 \mathrm{x}$ g for $30 \mathrm{~min}$ at $4^{\circ} \mathrm{C}$. The urinary sediments were recovered by discarding the supernatant, and total RNA was extracted using RiboZol reagent (Amresco LLC) according to the manufacturer's protocol. cDNA was synthesized by reverse transcription using $1 \mu \mathrm{g}$ total RNA and a QuantiTect Reverse Transcription kit according to the manufacturer's protocol (Qiagen, Inc.). The cDNA was used to perform quantitative PCR using EvaGreen 5x qPCR mix (qARTA Bio Inc.) and $10 \mathrm{pmol} / \mu \mathrm{l}$ each of the reverse and forward primers in a Lightcycler 96 (Roche Diagnostics). The sequences of the primers used to amplify human lncRNA TUG1 (30), nephrin (NSPH1), podocin (NSPH2), transcription factor A, mitochondrial (TFAM), cytochrome $\mathrm{C}$ oxidase subunit 5A (COX5A), PPARGC1A and GAPDH are shown in Table I. The amplification conditions consisted of 1 cycle of initial denaturation at $95^{\circ} \mathrm{C}$ for $15 \mathrm{~min}$; followed by 40 cycles of denaturation at $95^{\circ} \mathrm{C}$ for $15 \mathrm{sec}$, annealing at $56^{\circ} \mathrm{C}$ for $20 \mathrm{sec}$ and extension at $72^{\circ} \mathrm{C}$ for $20 \mathrm{sec}$. GAPDH was used as the internal control to normalize gene expression data. The relative mRNA expression was calculated using the $2^{-\Delta \Delta \mathrm{Cq}}$ method (31).

In silico identification of TUG1/miR-204-5p axis mRNA targets. The VarElect phenotype program from GeneAnalytics (geneanalytics.genecards.org), a pathway enrichment analysis tool, was used to identify predicted mRNA targets of the TUG1/miR-204-5p axis involved in mitochondrial biogenesis based on the genomic information stored in GeneCards (32). In total, 453 predicted mRNA targets of hsa-miR-204-5p downloaded from TargetScan version 7.2 (targetscan.org) (33) were profiled with GeneAnalytics. The entries were scored and ranked using an algorithm that enables matching of genomic expression, sequencing and microarray datasets to tissues and cells.

Statistical analysis. Data are expressed as the mean \pm standard error of the mean. Non-parametric variables were compared using a Mann-Whitney U-test or a Kruskal-Wallis test with a Dunn's post-hoc test, as appropriate. A Spearman's rank correlation analysis was used to analyse correlations. Statistical analysis was performed in GraphPad Prism Version 6.01 (GraphPad Software, Inc.). $\mathrm{P}<0.05$ was considered to indicate a statistically significant difference.

\section{Results}

Clinical and biochemical characteristics of glomerulonephritides patients and healthy controls. The present study included 
Table I. Sequences of the primers.

\begin{tabular}{llc}
\hline Gene & \multicolumn{1}{c}{ Sequence, 5'-3' } & Size, bp \\
\hline TUG1 & & 150 \\
Forward & TAGCAGTTCCCCAATCCTTG & \\
Reverse & CACAAATTCCCATCATTCCC & \\
NPHS1 & & 124 \\
Forward & GTGCACTATGCTCCCACCAT & \\
Reverse & TCTCCCAGTTGAACATGCCC & \\
NPHS2 & & 160 \\
Forward & GAGGAAGGTACCAAATCCTCCG & \\
Reverse & GCAGATGTCCCAGTCGGAATA & \\
TFAM & & 105 \\
Forward & ATGGAGGCAGGAGTTTCGTT & \\
Reverse & CCTAGATGAGTTCTGCCTGCT & \\
COX5A & & 160 \\
Forward & AGATGCCTGGGAATTGCGTA & \\
Reverse & AGGTCCTGCTTTGTCCTTAACA & \\
PPARGC1A & & \\
Forward & TGTGGAGTCCCTGGAATGGA & \\
Reverse & AAGATCGTGTTGGGCGAGAG & \\
GAPDH & & \\
Forward & CCCACTCCTCCACCTTTGAC & \\
Reverse & TGGTCCAGGGGTCTTACTCC & \\
\hline
\end{tabular}

10 patients with biopsy-confirmed diagnosis, 4 males and 6 females, and 10 sex-matched healthy controls. The mean age at renal biopsy was $34.4 \pm 11.1$ years old, the mean body mass index was $30.1 \pm 5.1$, and the diagnoses were as follows: 5 cases of FSGS, 3 cases of LN, 1 case of AAV and 1 case of advanced glomerular sclerosis (AGS). Table II shows the biochemical parameters of the glomerulonephritides patients. A total of $80 \%$ of the patients had haemoglobin values within the normal range. All glomerulonephritides patients had proteinuria, with the highest levels present in the patient diagnosed with AGS. However, CKD staging, classified according to the level of GFR (34) varied greatly. Only 2 patients had a normal GFR of $>90 \mathrm{ml} / \mathrm{min} / 1.73 \mathrm{~m}^{2} ; 3$ FSGS patients were Stage 2; 1 AAV patient was Stage $3 B ; 3$ patients were Stage 4 and only $1 \mathrm{LN}$ patient was Stage 5 with a GFR $<15 \mathrm{ml} / \mathrm{min} / 1.73 \mathrm{~m}^{2}$. The presence of glomerular damage in the absence of renal failure highlights the importance of identifying novel circulating markers able to detect glomerulonephritides during the early stages of CKD.

Glomerulonephritides patients have low levels of urinary TUGI expression. Cell-free IncRNAs are stable in urine and may potentially be used as non-invasive biomarkers to identify diseases, such as lupus nephritis and membranous nephropathy $(18,19)$. To assess alterations in TUG1 expression in the urinary sediment of patients with glomerulonephritides, RT-qPCR was used. Urinary expression of TUG1 was reduced in glomerulonephritides patients compared with the healthy controls (Fig. 1A). Furthermore, significantly lower TUG1 expression levels in urinary sediment was observed in patients with FSGS when compared with all
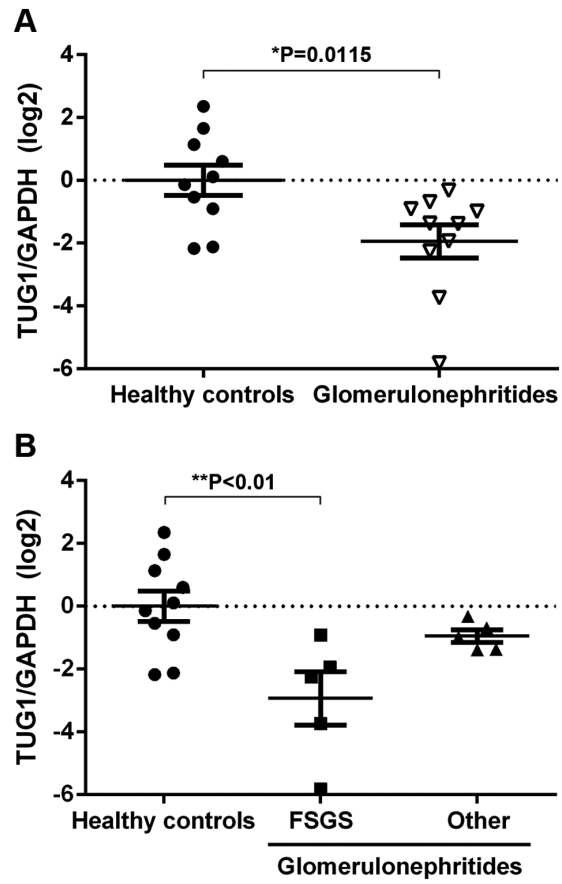

Figure 1. Urinary expression of TUG1 is decreased in FSGS patients (A) RT-qPCR results showing the $\log _{2}$-fold change in expression of TUG1/GAPDH in the urinary sediment of healthy controls $(n=10)$ and glomerulonephritides patients $(n=10)$. (B) Reverse transcription-quantitative PCR results showing the $\log _{2}$-fold change in expression of TUG1/GAPDH in the urinary sediment of healthy controls $(n=10)$, FSGS patients $(n=5)$ and other diagnoses (lupus nephritis, $n=3$; ANCA-associated vasculitis, $\mathrm{n}=1$; and advanced glomerular sclerosis, $\mathrm{n}=1$ ). Data are presented as the mean \pm standard error of the mean. ${ }^{*} \mathrm{P}<0.05,{ }^{* *} \mathrm{P}<0.01$. TUG1, taurine upregulated gene 1; FSGS, focal segmental glomerulosclerosis.

other diagnoses (Fig. 1B). These results suggest that the urinary expression of TUG1 decreases with podocytopathy.

Urinary expression of TUG1 correlates with podocyte marker expression. Glomerular injury is associated with podocyte loss, a phenomenon that can be monitored by detecting podocyte-specific mRNAs in the urine (35). In the present study, two podocyte-specific mRNAs, NPHS1 and NPHS2, were detected using RT-qPCR. Although the expression levels of urinary NPHS1 was not significantly different between glomerulonephritides patients and healthy controls, NPHS1 urinary expression was significantly lower in the FSGS patients when compared with other diagnoses (Fig. 2A). In contrast, NPHS2 mRNA did not differ significantly amongst the groups (Fig. 2B). Interestingly, a positive correlation was observed between TUG1 urinary expression and both podocyte-specific mRNAs (NPHS1, $r=0.6486,{ }^{* *} \mathrm{P}<0.01$; NPHS2, $\mathrm{r}=0.4782,{ }^{*} \mathrm{P}<0.05$; Fig. 2C and D). These results further suggest an association between TUG1 urinary expression and podocyte damage.

Urinary expression of mitochondrial biogenesis markers is correlated with TUG1 expression levels. Several studies have validated the functional role of TUG1 as a lncRNA in directly binding to specific miRNAs to regulate post-transcriptional processing via a competing endogenous RNA mechanism $(21,23,28,30)$. One such target is miR-204-5p (30). Thus, the VarElect phenotype program from Gene Analytics was used to predict mRNA targets of the TUG1/miR-204-5p 
Table II. Biochemical parameters of glomerulonephritides patients.

Patient no.

\begin{tabular}{|c|c|c|c|c|c|c|c|c|c|c|}
\hline Factor & 1 & 2 & 3 & 4 & 5 & 6 & 7 & 8 & 9 & 10 \\
\hline Diagnosis & FSGS & FSGS & AAV & LN & AGS & FSGS & LN & LN & FSGS & FSGS \\
\hline Hemoglobin, g/dl & 14.7 & 13.2 & 12.8 & 11.6 & 8.7 & 10.5 & 15.6 & 13.8 & 16.7 & 16.3 \\
\hline $\mathrm{sCr}, \mathrm{mg} / \mathrm{dl}$ & 0.84 & 0.57 & 1.5 & 3.7 & 3.9 & 2.1 & 0.86 & 8.4 & 1.4 & 1.5 \\
\hline $\mathrm{GFR}, \mathrm{ml} / \mathrm{min} / 1.73 \mathrm{~m}^{2}$ & 90 & 126 & 38 & 17 & 15 & 28 & 107 & 8 & 66 & 63 \\
\hline Proteinuria, g/24 h & 1.28 & 2.68 & 1.9 & 2.24 & 5.8 & 3.68 & 1.59 & 2.9 & 2.72 & 1.79 \\
\hline Albumin, g/dl & 3.7 & 2.1 & 4.1 & 3.6 & 2.5 & 2.3 & 2.9 & 3 & 3.5 & 4.3 \\
\hline pANCA & + & - & + & +++ & - & + & +++ & +++ & - & - \\
\hline cANCA & - & - & - & - & - & - & - & - & - & - \\
\hline Antinuclear antibodies & - & 0.09722 & 0.26389 & 0.48611 & 0.09722 & 0.09722 & 0.15278 & 0.15278 & 0.15278 & - \\
\hline Anti-DNA antibodies & - & - & - & +++ & - & - & ++ & ++ & - & - \\
\hline $\mathrm{C} 3$ fraction, mg/dl & 124 & 112 & 93.9 & 55 & 104 & 127 & 59.7 & 92.2 & 143 & 125 \\
\hline $\mathrm{C} 4$ fraction, mg/dl & 28.6 & 38.4 & 24.4 & 7 & 31.8 & 28.9 & 9.29 & 19.4 & 33.5 & 33.5 \\
\hline $\mathrm{CRP}, \mathrm{mg} / \mathrm{l}$ & 3.75 & $<3.23$ & 5.53 & $<3.23$ & 15.8 & $<3.23$ & 16 & 11.1 & $<3.23$ & $<3.23$ \\
\hline $\mathrm{ESR}, \mathrm{mm} / \mathrm{h}$ & 37 & 42 & 16 & 12 & 18 & - & 28 & 26 & - & 10 \\
\hline
\end{tabular}

FSGS, focal segmental glomerulosclerosis; LN, lupus nephritis; AVV, ANCA-associated vasculitis; AGS, advanced global sclerosis; sCr, serum creatinine; pANCA, perinuclear/nuclear antineutrophil cytoplasmic antibody; cANCA, cytoplasmic antineutrophil cytoplasmic antibody; C3, complement component 3; C4, complement component 4; CRP, C reactive protein; ESR, erythrocyte sedimentation rate; -, negative; + , low; ++, moderate; +++, high.

A

NPHS1
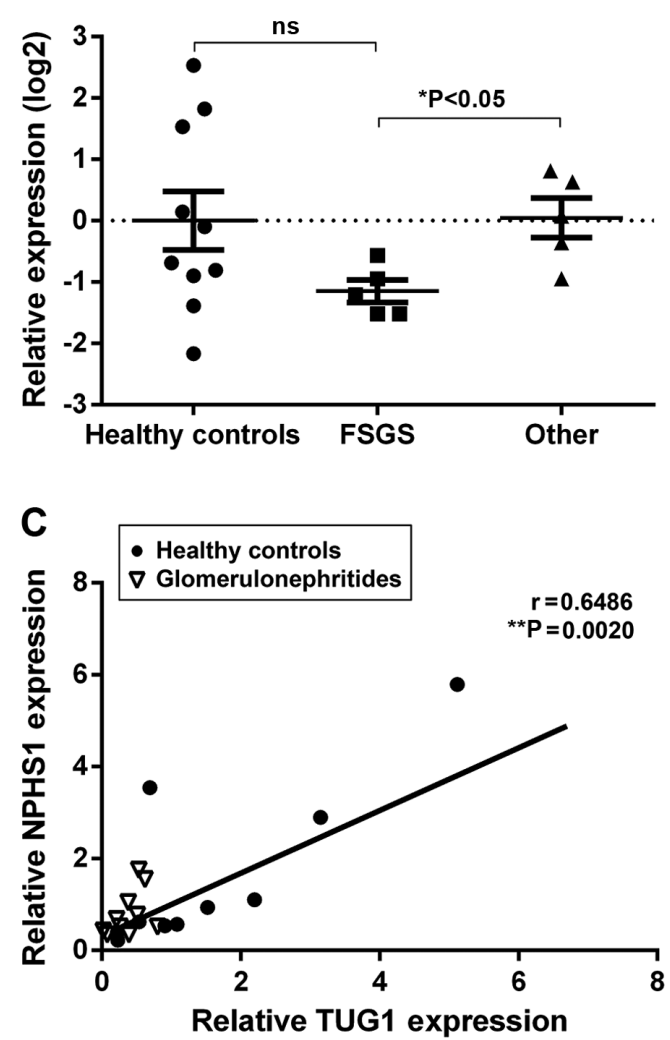

B

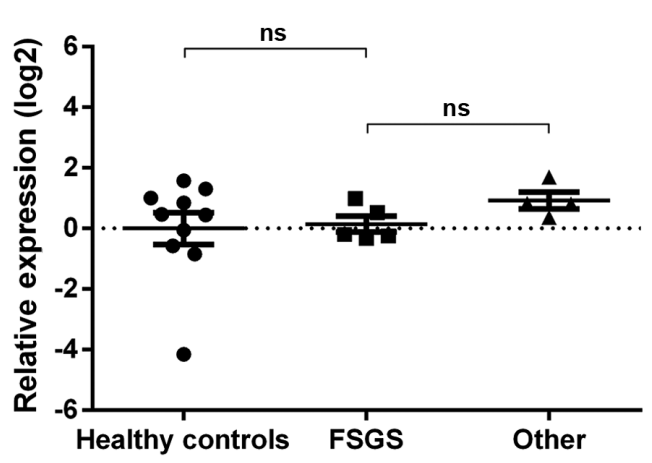

D

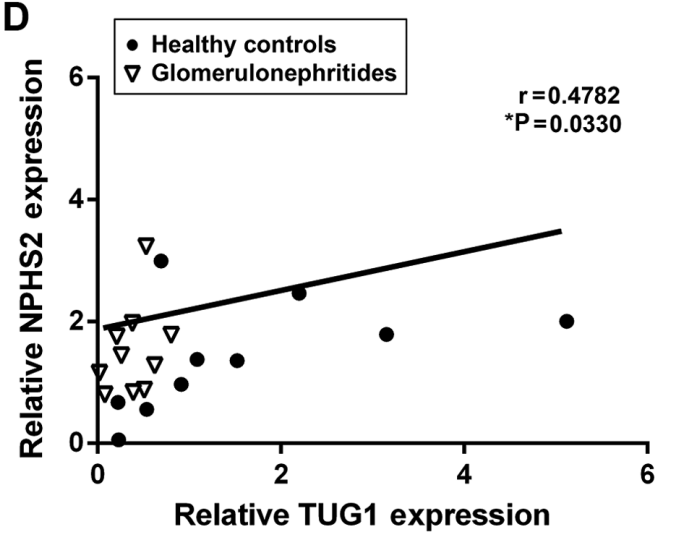

Figure 2. TUG1 urinary expression is correlated with podocyte marker mRNA expression in glomerulonephritides patients and healthy controls. Reverse transcription-quantitative PCR results showing the $\log _{2}$-fold change in expression of (A) NPHS1 and (B) NPHS2 in the urinary sediment of healthy controls $(n=10)$, FSGS patients $(n=5)$ and other diagnoses (lupus nephritis, $n=3$; ANCA-associated vasculitis, $n=1$; and advanced glomerular sclerosis, $n=1)$. Data are presented as the mean \pm standard error of the mean. ${ }^{*} \mathrm{P}<0.05$. Correlation between 1ncRNA TUG1 transcript levels and (C) NPHS1 or (D) NPHS2 in the urinary sediment of healthy controls $(n=10)$ and glomerulonephritides patients $(n=10)$. ${ }^{*}<0.05,{ }^{* *} \mathrm{P}<0.01$. TUG1, taurine upregulated gene 1 ; NSPH1, nephrin; NSPH2, podocin; ns, not significant. 
Table III. Predicted targets of TUG1/miR-204-5p axis involved in mitochondrial biogenesis.

\begin{tabular}{|c|c|c|c|}
\hline Symbol & Description & Global Rank ${ }^{\mathrm{a}}$ & Score ${ }^{a, b}$ \\
\hline PPARGC1A & PPARG Coactivator $1 \alpha$ & 1 & 18.76 \\
\hline TFAM & Transcription Factor A, Mitochondrial & 4 & 11.67 \\
\hline CREB1 & CAMP Responsive Element Binding Protein 1 & 25 & 4.98 \\
\hline SIRT1 & Sirtuin 1 & 26 & 4.80 \\
\hline COX5A & Cytochrome C Oxidase Subunit 5A & 39 & 3.13 \\
\hline ATF2 & Activating Transcription Factor 2 & 52 & 2.42 \\
\hline MAPK1 & Mitogen-Activated Protein Kinase 1 & 56 & 2.32 \\
\hline ESR1 & Estrogen Receptor 1 & 61 & 1.96 \\
\hline CAMK2D & Calcium/Calmodulin Dependent Protein Kinase II $\delta$ & 68 & 1.68 \\
\hline NFATC3 & Nuclear Factor of Activated T Cells 3 & 68 & 1.68 \\
\hline OGT & O-Linked N-Acetylglucosamine (GlcNAc) Transferase & 69 & 1.59 \\
\hline MXI1 & MAX Interactor 1, Dimerization Protein & 69 & 1.59 \\
\hline ESRRG & Estrogen Related Receptor $\gamma$ & 75 & 0.68 \\
\hline CPOX & Coproporphyrinogen Oxidase & 75 & 0.68 \\
\hline ESR2 & Estrogen Receptor 2 & 80 & 0.36 \\
\hline TFAP2A & Transcription Factor AP- $2 \alpha$ & 80 & 0.36 \\
\hline ZBTB20 & Zinc Finger and BTB Domain Containing 20 & 81 & 0.26 \\
\hline NPAS3 & Neuronal PAS Domain Protein 3 & 81 & 0.26 \\
\hline NR4A2 & Nuclear Receptor Subfamily 4 Group A Member 2 & 81 & 0.26 \\
\hline RICTOR & RPTOR Independent Companion of mTOR Complex 2 & 81 & 0.26 \\
\hline CAMK1 & Calcium/Calmodulin Dependent Protein Kinase I & 81 & 0.26 \\
\hline TET2 & Tet Methylcytosine Dioxygenase 2 & 81 & 0.26 \\
\hline ATXN1 & Ataxin 1 & 81 & 0.26 \\
\hline VHL & Von Hippel-Lindau Tumor Suppressor & 81 & 0.26 \\
\hline HMGCR & 3-Hydroxy-3-Methylglutaryl-CoA Reductase & 81 & 0.26 \\
\hline ZNF521 & Zinc Finger Protein 521 & 81 & 0.26 \\
\hline
\end{tabular}

FSGS, focal segmental glomerulosclerosis; LN, lupus nephritis; AVV, ANCA-associated vasculitis; AGS, advanced global sclerosis; sCr, serum creatinine; CRP, C reactive protein; ESR, erythrocyte sedimentation rate. ${ }^{\mathrm{a}}$ Obtained from a total of 453 genes. ${ }^{\mathrm{b}} \mathrm{Based}$ on analysis in VarElect of miR-204-5p predicted targets from TargetScan.

axis involved in mitochondrial biogenesis. Through gene data profiling, a total of 27 genes significantly associated with mitochondrial biogenesis were identified (Table III).

Next, the urinary expression of two predicted TUG1/miR-204 mRNA targets significantly associated with mitochondrial biogenesis, TFAM and COX5A, as well as one validated target, PPARGC1A (36), were quantified (Fig. 3). The relative expression levels of COXA5 and PPARGC1A were lower in the urinary sediment of glomerulonephritides patients when compared with healthy controls (Fig. 3C and E). Additionally, there was a significant correlation between TUG1 urinary expression and all three mitochondrial biogenesis mRNAs quantified in the present study (TFAM, $\mathrm{r}=0.6328,{ }^{* *} \mathrm{P}<0.01$; COXA5, $\mathrm{r}=0.4872,{ }^{*} \mathrm{P}<0.05$; PPARGC1A, $\mathrm{r}=0.7206,{ }^{* *} \mathrm{P}<0.01$; Fig. 3B-F). These results suggest that urinary RNAs may reflect molecular processes involved in the pathophysiology of renal dysfunction.

\section{Discussion}

The diagnosis of glomerulonephritides in current clinical practice is primarily dependent on the histological analysis of renal biopsies (6). However, this traditional approach is being challenged by molecular techniques that go beyond description and examine disease mechanisms (37,38). Multiple studies have shown the association between dysregulated expression of certain lncRNAs and the development and progression of pathological disease states that affect the kidney (12-17). Urinary non-coding RNAs are a promising non-invasive tool able to reflect renal disease, aid in its appropriate diagnosis, and guide therapeutic choices $(12,39,40)$. In the present study, it was shown that IncRNA TUG1 was present in the urinary sediment, and its expression was significantly reduced in patients with biopsy-confirmed glomerulonephritides, particularly those diagnosed with FSGS. Moreover, there was a positive correlation between urinary TUG1 expression and mRNAs known to be involved in pathogenic mechanisms associated with podocyte loss.

Mutations in NPHS1 or NPHS2 lead to proteinuria and rapid ESRD progression, highlighting the critical role of these podocyte-specific structural proteins in glomerular filtration barrier function $(41,42)$. Although NPHS1 and NPHS2 are detectable in the urine of glomerulonephritides patients and 
A

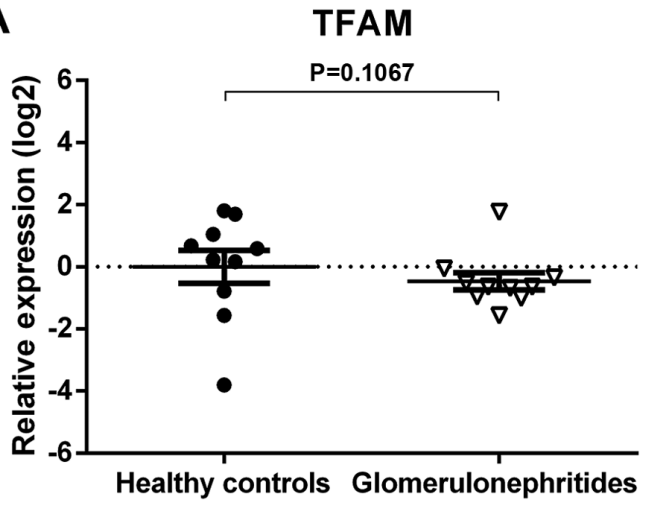

C

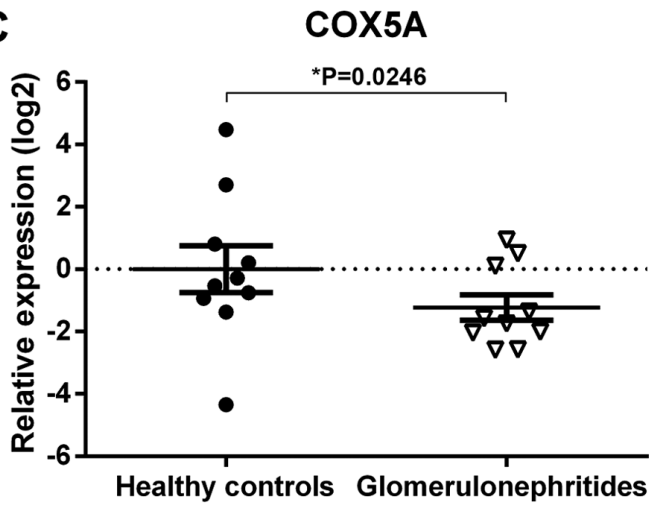

E

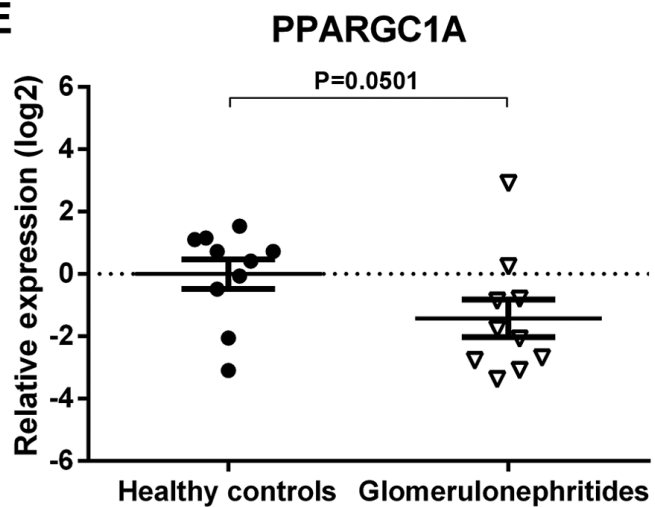

B

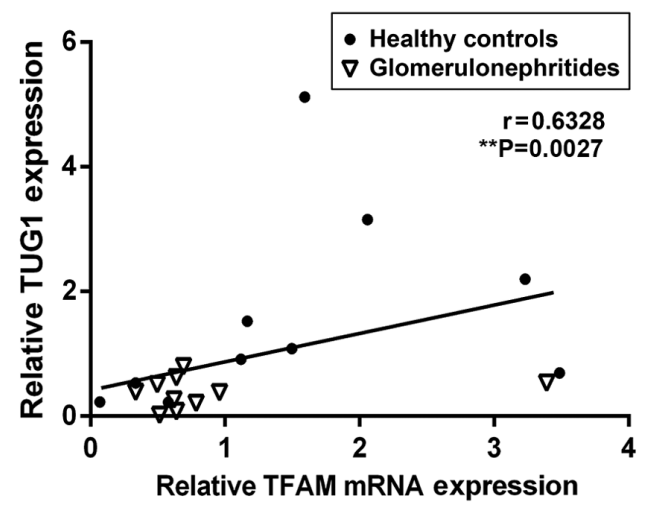

$\mathbf{D}$

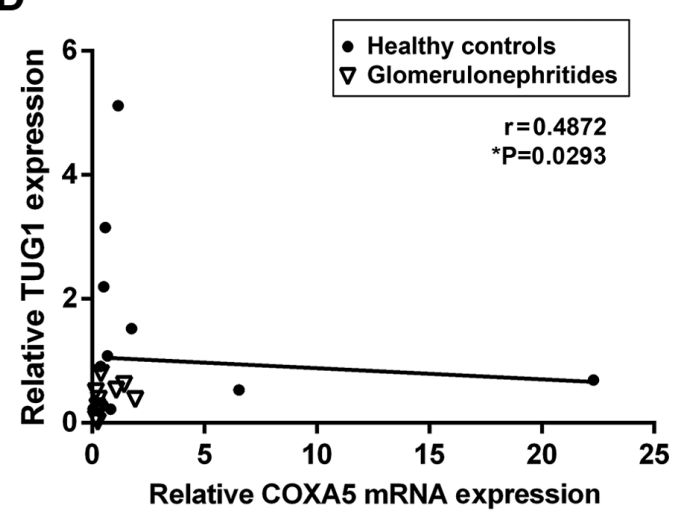

$\mathbf{F}$

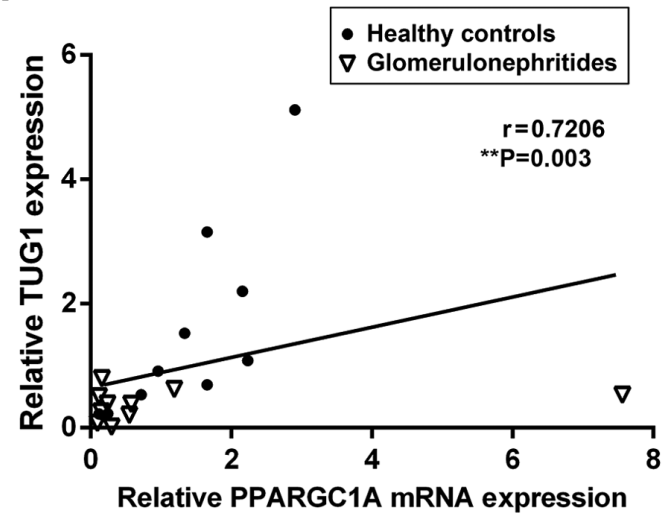

Figure 3. Levels of urinary lncRNA TUG1 correlate with mitochondrial biogenesis mRNAs predicted to be modulated by the TUG1/miR-2014-5p axis. Reverse transcription-quantitative PCR results showing the $\log _{2}$-fold change in expression of (A) TFAM, (C) COX5A and (E) PPARGC1A in the urinary sediment of healthy controls $(n=10)$ and glomerulonehritides patients $(n=10)$. Data are presented as the mean \pm standard error of the mean. Correlation between IncRNA TUG1 transcript levels and (B) TFAM, (D) COXA5 and (F) PARGC1A in the urinary sediment of healthy controls (n=10) and glomerulonephritides patients $(\mathrm{n}=10)$. ${ }^{*} \mathrm{P}<0.05,{ }^{* *} \mathrm{P}<0.01$. TUG1, taurine upregulated gene 1; miR, microRNA; TFAM, transcription factor A mitochondrial, COX5A, cytochrome C oxidase subunit 5A; and PPARGC1A, peroxisome proliferator-activated receptor $\gamma$ coactivator $1 \alpha$.

have been described as clinically valuable biomarkers of podocyte injury, the results between studies are inconsistent and raise questions regarding the reasons behind the contrasting findings (43). In the present study, a decrease in urinary NPHS1 expression in FSGS was observed when compared to other diagnoses, but no differences in NPHS2 levels within the groups was found. These results partially agree with previous studies that showed urinary NPHS1 and NPHS2 mRNA levels were reduced in patients with MCN and FSGS, a feature that correlates with the degree of proteinuria $(44,45)$. However, TUG1 expression levels showed no correlation with GFR, proteinuria or albuminuria in the present study (data not shown). Although it has been proposed that these podocyte-specific biomarkers can detect nephropathy before the development of albuminuria (46-48), a larger patient cohort is required to assess the relationship between TUG1 urinary expression and clinical markers of renal dysfunction traditionally used in the diagnosis of glomerulonephritides. In the present study, a positive correlation between NPHS1 and NPHS2 mRNA levels and TUG1 expression was observed, which suggests that the molecular mechanisms described in vitro and in animal models of DN regarding the relationship between TUG1 and podocyte loss are reflected in the urine of non-diabetic patients (28). 
A decrease in PGC1A, a transcriptional coactivator that regulates energy homeostasis and mitochondrial biogenesis, has been implicated in the development of acute kidney injury, DN and renal fibrosis (49). The expression levels and activity of PGC1A depend on multiple transcriptional and post-transcriptional mechanisms, some mediated by non-coding RNAs $(22,28)$. Interactions between TUG1 and the promoter of PGC1A enhances its transcription, and increases mitochondrial content and cellular ATP levels whilst reducing mitochondrial reactive oxygen species levels. In addition, TUG1 acts as a competitive endogenous RNA for miRNAs such as miR-145, miR-144 and miR-204-5p $(30,50,51)$. miRNA miR-204-5p is involved in multiple cellular processes, including angiogenesis, vascular disease, metabolism and glucose homeostasis $(30,52)$. Through in silico analysis, targets of the TUG1/miR-204-5p axis involved in mitochondrial biogenesis were predicted and it was subsequently shown that PGC1A, COX5A and TFAM were also detectable in the urine and were correlated with TUG1 expression. PGC1A is a known posttranscriptional target of miR-204-5p $(53,54)$; however, COX5A and TFAM still require experimental validation. Nonetheless, the results suggest that the TUG1/miR-204 axis and their mitochondrial biogenesis targets are relevant in FSGS pathogenesis and may serve as potential biomarkers.

The present study has some limitations, including the small number of glomerulonephritides patients enrolled and the variability of diagnosis, which included primary and secondary glomerulonephritides. Nonetheless, our findings support a role for lncRNA TUG1 in the development of glomerulonephritides, and justify further exploration of urinary TUG1 as a potential biomarker of FSGS. More extensive studies, with carefully selected subjects are required to confirm the relationship between urinary TUG1 expression, clinical markers of renal dysfunction and glomerular damage observed in kidney biopsies; and to validate lncRNA TUG1 as a biomarker of FSGS. Additionally, more in vitro and animal experiments focused on the TUG1/miR-204-5p axis should be performed to increase our understanding of these non-coding RNAs in mitochondrial bioenergetics and podocyte loss.

In conclusion, the results of the present study highlight the potential of urinary non-coding RNAs, such as TUG1, in the diagnosis of renal disease, and suggests that this lncRNA and its mitochondrial-associated pathways are relevant in glomerulonephritides other than DN. Further studies are required to evaluate urinary TUG1 as a potential biomarker of podocytopathy, and to determine its association with kidney dysfunction and patient prognosis.

\section{Acknowledgements}

We would like to thank Claudia Susana Meza Calvillo for her technical assistance as part of the Scientific Research Summer Program of the Mexican Academy of Science 2019.

\section{Funding}

This study was supported by Consejo Nacional de Ciencia y Tecnología (CONACyT), Mexico (grant no. SALUD-201802-B-S-42687).

\section{Availability of data and materials}

The datasets used and/or analysed during the present study are available from the corresponding author on reasonable request.

\section{Authors' contributions}

FJS-T, MM-P and RE conceived the study. FJS-T and MM-P collected and analysed the data. ZM performed the experiments. CM-C performed the histopathological analysis of renal biopsies. RE drafted the manuscript. All authors read and approved the final manuscript.

\section{Ethics approval and consent to participate}

The present study complied with the ethical principles for medical research specified in the Declaration of Helsinki and was approved by the Local Ethics and Research Committee at the Hospital de Especialidades NUM. 1, Bajio, Leon, Guanajuato, Instituto Mexicano del Seguro Social (approval no. CLIEIS R-2018-1001-114). Each participant provided written informed consent prior to enrolment in the study.

\section{Patient consent for publication}

Not applicable.

\section{Competing interests}

The authors declare that they have no competing interests.

\section{References}

1. Floege $\mathrm{J}$ and Amann K: Primary glomerulonephritides. Lancet 387: 2036-2048, 2016.

2. Caliskan Y and Kiryluk K: Novel biomarkers in glomerular disease. Adv Chronic Kidney Dis 21: 205-216, 2014.

3. Matovinović MS: Podocyte injury in glomerular diseases. EJIFCC 20: 21-27, 2009.

4. Mallipattu SK and He JC: The podocyte as a direct target for treatment of glomerular disease? Am J Physiol Renal Physiol 311: F46-F51, 2016.

5. Bagavant $\mathrm{H}$ and Fu SM: Pathogenesis of kidney disease in systemic lupus erythematosus. Curr Opin Rheumatol 21: 489-494, 2009.

6. Hogan J, Mohan P and Appel GB: Diagnostic tests and treatment options in glomerular disease: 2014 update. Am J Kidney Dis 63: 656-666, 2014

7. Parikh SV, Ayoub I and Rovin BH: The kidney biopsy in lupus nephritis: Time to move beyond histology. Nephrol Dial Transplant 30: 3-6, 2015.

8. Rovin BH, Almaani S and Malvar A: Reimagining the kidney biopsy in the era of diagnostic biomarkers of glomerular disease. Kidney Int 95: 265-267, 2019.

9. Kopp F and Mendell JT: Functional classification and experimental dissection of long noncoding RNAs. Cell 172: 393-407, 2018.

10. Mongelli A, Martelli F, Farsetti A and Gaetano C: The dark that matters: Long Non-coding RNAs as master regulators of cellular metabolism in Non-communicable diseases. Front Physiol 10: 369, 2019.

11. Lorenzen JM and Thum T: Long noncoding RNAs in kidney and cardiovascular diseases. Nat Rev Nephrol 12: 360-373, 2016.

12. Ignarski M, Islam R and Müller RU: Long Non-coding RNAs in kidney disease. Int J Mol Sci 20: 3276, 2019.

13. Tian H, Wu M, Zhou P, Huang C, Ye C and Wang L: The long non-coding RNA MALAT1 is increased in renal ischemiareperfusion injury and inhibits hypoxia-induced inflammation. Ren Fail 40: 527-533, 2018 
14. Hu S, Han R, Shi J, Zhu X, Qin W, Zeng C, Bao H and Liu Z: The long noncoding RNA LOC 105374325 causes podocyte injury in individuals with focal segmental glomerulosclerosis. J Biol Chem 293: 20227-20239, 2018.

15. Han R, Hu S, Qin W, Shi J, Zeng C, Bao H and Liu Z: Upregulated long noncoding RNA LOC105375913 induces tubulointerstitial fibrosis in focal segmental glomerulosclerosis. Sci Rep 9: 716, 2019.

16. Jin LW, Pan M, Ye HY, Zheng Y, Chen Y, Huang WW, Xu XY and Zheng SB: Down-regulation of the long non-coding RNA XIST ameliorates podocyte apoptosis in membranous nephropathy via the miR-217-TLR4 pathway. Exp Physiol 104: 220-230, 2019.

17. Liao Z, Ye Z, Xue Z, Wu L, Ouyang Y, Yao C, Cui C, Xu N, Ma J, Hou G, et al: Identification of renal long Non-coding RNA RP11-2B6.2 as a positive regulator of type I interferon signaling pathway in lupus nephritis. Front Immunol 10: 975, 2019.

18. Santer L, López B, Ravassa S, Baer C, Riedel I, Chatterjee S, Moreno MU, González A, Querejeta R, Pinet F, et al: Circulating long noncoding RNA LIPCAR predicts heart failure outcomes in patients without chronic kidney disease. Hypertension 73: 820-828, 2019

19. Huang YS, Hsieh HY, Shih HM, Sytwu HK and Wu CC: Urinary $\mathrm{Xist}$ is a potential biomarker for membranous nephropathy. Biochem Biophys Res Commun 452: 415-421, 2014

20. Forbes JM and Thorburn DR: Mitochondrial dysfunction in diabetic kidney disease. Nat Rev Nephrol 14: 291-312, 2018.

21. Young TL, Matsuda T and Cepko CL: The noncoding RNA taurine upregulated gene 1 is required for differentiation of the murine retina. Curr Biol 15: 501-512, 2005.

22. Li SY, Park J, Qiu C, Han SH, Palmer MB, Arany Z and Susztak K: Increasing the level of peroxisome proliferator-activated receptor $\gamma$ coactivator- $1 \alpha$ in podocytes results in collapsing glomerulopathy. JCI Insight 2: e92930, 2017.

23. Li SY and Susztak K: The long noncoding RNA Tug1 connects metabolic changes with kidney disease in podocytes. J Clin Invest 126: 4072-4075, 2016.

24. LeBleu VS, O'Connell JT, Gonzalez Herrera KN, Wikman H, Pantel K, Haigis MC, de Carvalho FM, Damascena A, Domingos Chinen LT, Rocha RM, et al: PGC-1 $\alpha$ mediates mitochondrial biogenesis and oxidative phosphorylation in cancer cells to promote metastasis. Nat Cell Biol 16: 992-1003, 1-15, 2014.

25. Uldry M, Yang W, St-Pierre J, Lin J, Seale P and Spiegelman BM: Complementary action of the PGC-1 coactivators in mitochondrial biogenesis and brown fat differentiation. Cell Metab 3 : 333-341, 2006.

26. Finck BN and Kelly DP: PGC-1 coactivators: Inducible regulators of energy metabolism in health and disease. J Clin Invest 116 : 615-622, 2006

27. Kang HM, Ahn SH, Choi P, Ko YA, Han SH, Chinga F, Park AS Tao J, Sharma K, Pullman J, et al: Defective fatty acid oxidation in renal tubular epithelial cells has a key role in kidney fibrosis development. Nat Med 21: 37-46, 2015.

28. Long J, Badal SS, Ye Z, Wang Y, Ayanga BA, Galvan DL, Green NH, Chang BH, Overbeek PA and Danesh FR: Long noncoding RNA Tug1 regulates mitochondrial bioenergetics in diabetic nephropathy. J Clin Invest 126: 4205-4218, 2016.

29. Association WM: World medical association declaration of Helsinki: Ethical principles for medical research involving human subjects. JAMA 310: 2191-2194, 2013.

30. Yu C, Li L, Xie F, Guo S, Liu F, Dong N and Wang Y: LncRNA TUG1 sponges miR-204-5p to promote osteoblast differentiation through upregulating Runx 2 in aortic valve calcification. Cardiovasc Res 114: 168-179, 2018.

31. Livak KJ and Schmittgen TD: Analysis of relative gene expression data using real-time quantitative PCR and the 2(-Delta Delta C(T)) method. Methods 25: 402-408, 2001.

32. Ben-Ari Fuchs S, Lieder I, Stelzer G, Mazor Y, Buzhor E, Kaplan S, Bogoch Y, Plaschkes I, Shitrit A, Rappaport N, et al: GeneAnalytics: An integrative gene set analysis tool for next generation sequencing, RNAseq and microarray data. OMICS 20: 139-151, 2016.

33. Agarwal V, Bell GW, Nam JW and Bartel DP: Predicting effective microRNA target sites in mammalian mRNAs. Elife 4 : e05005, 2015.

34. Levey AS, Eckardt KU, Tsukamoto Y, Levin A, Coresh J, Rossert J, De Zeeuw D, Hostetter TH, Lameire N and Eknoyan G: Definition and classification of chronic kidney disease: A position statement from Kidney Disease: Improving Global Outcomes (KDIGO). Kidney Int 67: 2089-2100, 2005.
35. Fukuda A, Wickman LT, Venkatareddy MP, Wang SQ, Chowdhury MA, Wiggins JE, Shedden KA and Wiggins RC: Urine podocin:nephrin mRNA ratio (PNR) as a podocyte stress biomarker. Nephrol Dial Transplant 27: 4079-4087, 2012.

36. Civelek M, Hagopian R, Pan C, Che N, Yang WP, Kayne PS Saleem NK, Cederberg H, Kuusisto J, Gargalovic PS, et al: Genetic regulation of human adipose microRNA expression and its consequences for metabolic traits. Hum Mol Genet 22: 3023-3037, 2013.

37. Saez-Rodriguez J, Rinschen MM, Floege J and Kramann R: Big science and big data in nephrology. Kidney Int 95: 1326-1337, 2019.

38. Kretzler M, Cohen CD, Doran P, Henger A, Madden S, Gröne EF, Nelson PJ, Schlöndorff D and Gröne HJ: Repuncturing the renal biopsy: Strategies for molecular diagnosis in nephrology. J Am Soc Nephrol 13: 1961-1972, 2002.

39. Sun IO and Lerman LO: Urinary microRNA in kidney disease: Utility and roles. Am J Physiol Renal Physiol 316: F785-F793, 2019.

40. Brandenburger T, Salgado Somoza A, Devaux Y and Lorenzen JM: Noncoding RNAs in acute kidney injury. Kidney Int 94: 870-881, 2018.

41. Patrakka J, Kestilä M, Wartiovaara J, Ruotsalainen V, Tissari P, Lenkkeri U, Männikkö M, Visapää I, Holmberg C, Rapola J, et al: Congenital nephrotic syndrome (NPHS1): Features resulting from different mutations in Finnish patients. Kidney Int 58: 972-980, 2000

42. Boute N, Gribouval O, Roselli S, Benessy F, Lee H, Fuchshuber A, Dahan K, Gubler MC, Niaudet P and Antignac C: NPHS2, encoding the glomerular protein podocin, is mutated in autosomal recessive steroid-resistant nephrotic syndrome. Nat Genet 24: 349-354, 2000.

43. Sekulic M and Pichler Sekulic S: A compendium of urinary biomarkers indicative of glomerular podocytopathy. Patholog Res Int 2013: 782395, 2013.

44. Hara M, Yanagihara T and Kihara I: Urinary podocytes in primary focal segmental glomerulosclerosis. Nephron 89: 342-347, 2001.

45. Szeto CC, Wang G, Chow KM, Lai FM, Ma TK, Kwan BC, Luk CC and Li PK: Podocyte mRNA in the urinary sediment of minimal change nephropathy and focal segmental glomerulosclerosis. Clin Nephrol 84: 198-205, 2015.

46. Pätäri A, Forsblom C, Havana M, Taipale H, Groop PH and Holthöfer H: Nephrinuria in diabetic nephropathy of type 1 diabetes. Diabetes 52: 2969-2974, 2003.

47. Ng DP, Tai BC, Tan E, Leong H, Nurbaya S, Lim XL, Chia KS, Wong CS, Lim WY and Holthöfer H: Nephrinuria associates with multiple renal traits in type 2 diabetes. Nephrol Dial Transplant 26: 2508-2514, 2011.

48. Chang JH, Paik SY, Mao L, Eisner W, Flannery PJ, Wang L, Tang Y, Mattocks N, Hadjadj S, Goujon JM, et al: Diabetic kidney disease in FVB/NJ Akita mice: Temporal pattern of kidney injury and urinary nephrin excretion. PLoS One 7: e33942, 2012.

49. Lynch MR, Tran MT and Parikh SM: PGC1 $\alpha$ in the kidney. Am J Physiol Renal Physiol 314: F1-F8, 2018.

50. Tan J, Qiu K, Li M and Liang Y: Double-negative feedback loop between long non-coding RNA TUG1 and miR-145 promotes epithelial to mesenchymal transition and radioresistance in human bladder cancer cells. FEBS Lett 589: 3175-3181, 2015.

51. Cai H, Xue Y, Wang P, Wang Z, Li Z, Hu Y, Li Z, Shang X and Liu Y: The long noncoding RNA TUG1 regulates blood-tumor barrier permeability by targeting miR-144. Oncotarget 6 : 19759-19779, 2015.

52. Flores-Pérez A,MarchatLA, Rodríguez-Cuevas S, Bautista-PiñaV, Hidalgo-Miranda A, Ocampo EA, Martínez M, Palma-Flores C, Fonseca-Sánchez MA, Astudillo-de la Vega $\mathrm{H}$, et al: Dual targeting of ANGPT1 and TGFBR2 genes by miR-204 controls angiogenesis in breast cancer. Sci Rep 6: 34504, 2016.

53. Maniyadath B, Chattopadhyay T, Verma S, Kumari S, Kulkarni P, Banerjee K, Lazarus A, Kokane SS, Shetty T, Anamika K and Kolthur-Seetharam U: Loss of hepatic oscillatory fed microRNAs Abrogates Refed transition and causes liver dysfunctions. Cell Rep 26: 2212-2226, 2019

54. Houzelle A, Dahlmans D, Nascimento EBM, Schaart G, Jörgensen JA, Moonen-Kornips E, Kersten S, Wang X and Hoeks J: MicroRNA-204-5p modulates mitochondrial biogenesis in $\mathrm{C} 2 \mathrm{C} 12$ myotubes and associates with oxidative capacity in humans. J Cell Physiol 235: 9851-9863, 2020 